\title{
Separation and Characterization of Sulfated Glycopeptides from Ovomucin, Chalazae and Yolk Membrane in Chicken Eggs
}

\author{
Akio Kato, Yoshiharu Miyoshi, Masataka Suga \\ and Kunihiko KoBAYASHI \\ Department of Agricultural Chemistry, Faculty of Agriculture, \\ Yamaguchi University, Yamaguchi 753, Japan
}

Received November 9, 1981

\begin{abstract}
The sulfated glycopeptides in ovomucin, chalazae and yolk membrane were isolated from the proteolytic digests by gel filtration on a Bio-Gel P-100 column and DEAE-Sephadex A-25 column chromatography. These sulfated glycopeptides contained $N$-acetylhexosamine $(23.3 \sim 26.8 \%)$, hexose $(23.6 \sim 24.4 \%)$, sialic acid $(11.2 \sim 18.0 \%)$, sulfate $(5 \sim 12.1 \%)$ and peptide $(17.5 \sim 18.1 \%)$. The sulfate contents of glycopeptides in chalazae and yolk membrane were much higher than those in ovomucin, about two times in a molar ratio to hexosamine. The sedimentation patterns of each sulfated glycopeptide were single and the sedimentation constants were around $3 \mathrm{~S}$, suggesting that these sulfated glycopeptides were macromolecular components. Thus, the presence of highly sulfated glycoproteins was confirmed in chalazae and yolk membrane, which were different from those in ovomucin.
\end{abstract}

The yolk membrane and chalazae serve to stabilize the position of the yolk in the center of chicken egg. It is well-known that the yolk membrane and chalazae of chicken egg contain ovomucin-like glycoproteins. Feeney $e t$ al. ${ }^{1)}$ investigated the distribution of sialic acid in chicken egg white and found that the sialic acid contents of chalazae and yolk membrane were similar to ovomucin. From the results, they supported the hypothesis that the chalazae might be formed from ovomucin by a physical winding or twisting action and that the yolk membrane was supported by ovomucin layer. Sato and $\mathrm{Kato}^{2)}$ compared the carbohydrate rich and poor components of chalazae with those of ovomucin. They suggested that the carbohydrate-rich component of chalazae was slightly different from that of ovomucin in its carbohydrate and amino acid compositions and electrophoretic patterns, while the carbohydrate poor component of chalazae was the same as that of ovomucin. Kido et $a l .{ }^{3)}$ isolated three glycoproteins from yolk membranes and determined their chemical compositions. However, it is still not com- pletely elucidated whether chalazae and yolk membrane are composed of ovomucin itself or other glycoproteins. Since ovomucin has been reported to be a sulfated glycoprotein, the chalazae and yolk membrane also should be characterized as sulfated glycoproteins.

This paper describes the properties of the sulfated glycopeptide obtained from the proteolytic digests of chalazae, yolk membrane and ovomucin to compare these three structural constituents in chicken egg white.

\section{MATERIALS AND METHODS}

Preparation of ovomucin, chalazae and yolk membrane. All eggs were collected from hens within $24 \mathrm{hr}$ after laying. Thick white was separated from thin white using a sieve. Chalazaes in thick white were removed completely. The fresh thick white was separated into a gel-like precipitate and supernatant by ultracentrifugation for $60 \mathrm{~min}$ at $59,000 \times g$. The precipitate was washed with $2 \% \mathrm{KCl}$ solution without diluting with water until the washings were free from proteins. The precipitate was finally washed with water until the washings contained no chloride. The gel-like precipitates thus obtained were used as ovomucin. Chalazaes were separated from egg white and washed repeatedly with $2 \% \mathrm{KCl}$ solution until the washings were 
free from proteins. Yolk membrane was separated from egg yolk which carefully removed extraneous thick white and chalazae. A portion of yolk membranes were ruptured to squeeze out yolk. The resulting membranes were repeatedly washed by $2 \% \mathrm{KCl}$ solution until the washings were free from proteins.

Isolation of sulfated glycopeptides from ovomucin, chalazae and yolk membrane. Ovomucin, chalazae and yolk membrane were digested with pronase (Kaken Chemical Co.) at $30^{\circ} \mathrm{C}$ for $72 \mathrm{hr}$ in $0.05 \mathrm{M}$ Tris- $\mathrm{HCl}$ buffer, $\mathrm{pH} 8.0$. The digests were subsequently digested with papain (Katayama Chemical Industries Co.) at $30^{\circ} \mathrm{C}$ for $72 \mathrm{hr}$ in $0.05 \mathrm{M}$ Tris- $\mathrm{HCl}$ buffer, $\mathrm{pH} 7.0$, containing $0.01 \mathrm{M}$ cysteine and $0.002 \mathrm{M}$ EDTA. Action of proteolytic enzymes was performed with the addition of $0.05 \%$ sodium azide to protect from microorganisms and enzymes $(20 \mathrm{mg} / \mathrm{g}$ dry substrates) every $24 \mathrm{hr}$ under moderate stirring.

The proteolytic digests thus obtained were fractionated on a column $(1.8 \times 120 \mathrm{~cm})$ of Bio-Gel P-100, eluting with $0.5 \mathrm{M} \mathrm{NaCl}$. Effluents were collected in each fraction of $3 \mathrm{ml}$ and appropriate aliquots were analyzed for protein (absorbance at $280 \mathrm{~nm}$ ) and hexose (absorbance at $520 \mathrm{~nm}$ in the orcinol test). The void volume fraction was collected and then fractionated on a column of DEAE-Sephadex A25 , equilibrating with $0.1 \mathrm{M}$ pyridine-acetate buffer, $\mathrm{pH}$ 5.0. Elution was carried out with a linear gradient of $0.1 \sim 1.0 \mathrm{M}$ pyridine-acetate buffer, $\mathrm{pH} 5.0$. Effluents were collected in each fraction of $3 \mathrm{ml}$ and appropriate aliquots were analyzed for protein and hexose. Carbohydratecontaining fractions were collected and analyzed.

Analytical methods. Hexose content was determined by the orcinol method. ${ }^{4}$ Galactose and mannose were determined by gas-liquid chromatography, as described in the previous paper. ${ }^{5)}$ Total hexosamine was determined by the Elson-Morgan method, as modified by Neuhaus and Leutzring, $\left.{ }^{6}\right)$ in which an equimolar mixture of galactosamine and glucosamine was used as a standard. Samples were hydrolyzed with $3 \mathrm{~N} \mathrm{HCl}$ at $100^{\circ} \mathrm{C}$ for $4 \mathrm{hr}$ in sealed vacuum ampoules. After hydrolysis, the hydrolyzates were evaporated to dryness. Galactosamine and glucosamine were determined using a Hitachi KLA-3B amino acid analyzer. The hexosamines were represented as $N$-acetyl derivatives. Sialic acid content was determined by the thiobarbituric acid method ${ }^{7)}$ after hydrolysis of samples with $0.05 \mathrm{~N} \mathrm{H}_{2} \mathrm{SO}_{4}$ at $75^{\circ} \mathrm{C}$ for $1 \mathrm{hr}$ in sealed ampoules. Sulfate content was determined by the benzidine method ${ }^{8)}$ after hydrolysis of samples with $6 \mathrm{~N} \mathrm{HCl}$ at $100^{\circ} \mathrm{C}$ for $9 \mathrm{hr}$ in sealed ampoules. Amino acid analysis was performed on samples hydrolyzed with $6 \mathrm{~N} \mathrm{HCl}$ at $105^{\circ} \mathrm{C}$ for $24 \mathrm{hr}$ in sealed vacuum ampoules. After hydrolysis, the samples were evaporated to dryness and dissolved in $0.2 \mathrm{M}$ sodium citrate buffer, $\mathrm{pH} 2.2$, and then analyzed with a Hitachi KLA-3B amino acid analyzer.

Infrared absorption was recorded by a Hitachi EPI-B21 type infrared spectrophotometer, using $\mathrm{KBr}$ disc tech- nique. The samples were prepared by drying exhaustively in vacuo the freeze-dried mixture of $100 \mathrm{mg} \mathrm{KBr}$ and $1 \mathrm{mg}$ of sulfated glycopeptides over phosphorus pentoxide.

Ultracentrifugal analysis was carried out in a Hitachi Model UCA-1A analytical ultracentrifuge with Schlieren optics at $60,000 \mathrm{rpm}$ at $20^{\circ} \mathrm{C}$. Sample concentration was $5 \mathrm{mg} / \mathrm{ml}$ in $0.05 \mathrm{M}$ Tris- $\mathrm{HCl}$ buffer, $\mathrm{pH} 8.0$. Photographs were taken at $10 \mathrm{~min}$ intervals after reaching maximum speed.

\section{RESULTS AND DISCUSSION}

Table I shows the chemical composition of ovomucin, chalazae and yolk membrane. Sulfate residues were contained not only in ovomucin but also in chalazae and yolk membrane. It was difficult to purify the intact sulfated glycoproteins from these structural constituents in eggs, because of their insolubility and complexity. Generally, the sulfated glycoproteins of epithelial mucins and cellular membranes can be isolated from their proteolytic digests. Therefore, the sulfated glycoproteins of ovomucin, chalazae and yolk membrane were also isolated from the proteolytic digests. It seems reasonable to name sulfated glycopeptides instead of sulfated glycoproteins because of preparation from the proteolytic digests.

Figure 1 shows the elution patterns of the proteolytic digests of ovomucin, chalazae and yolk membrane by gel filtration on a Bio-Gel P-100 column. Two glycopeptide fractions were separated in the similar pattern that most glycopeptide fractions emerged in the void

TABle I. Carbohydrate Composition of Ovomucin, Chalazae AND YolK Membrane

\begin{tabular}{lccc}
\hline & \multicolumn{3}{c}{ Contents $(\mathrm{g} / 100 \mathrm{~g}$ dry materials $)$} \\
\cline { 2 - 4 } & Ovomucin & $\begin{array}{c}\text { Yolk } \\
\text { membrane }\end{array}$ & Chalazae \\
\hline $\begin{array}{l}N \text {-Acetyl- } \\
\text { hexosamine }\end{array}$ & 12.8 & 9.9 & 10.9 \\
$\begin{array}{l}\text { Hexose } \\
\text { Sialic acid }\end{array}$ & 12.5 & 7.7 & 6.8 \\
Sulfate & 9.9 & 3.2 & 3.6 \\
\hline
\end{tabular}




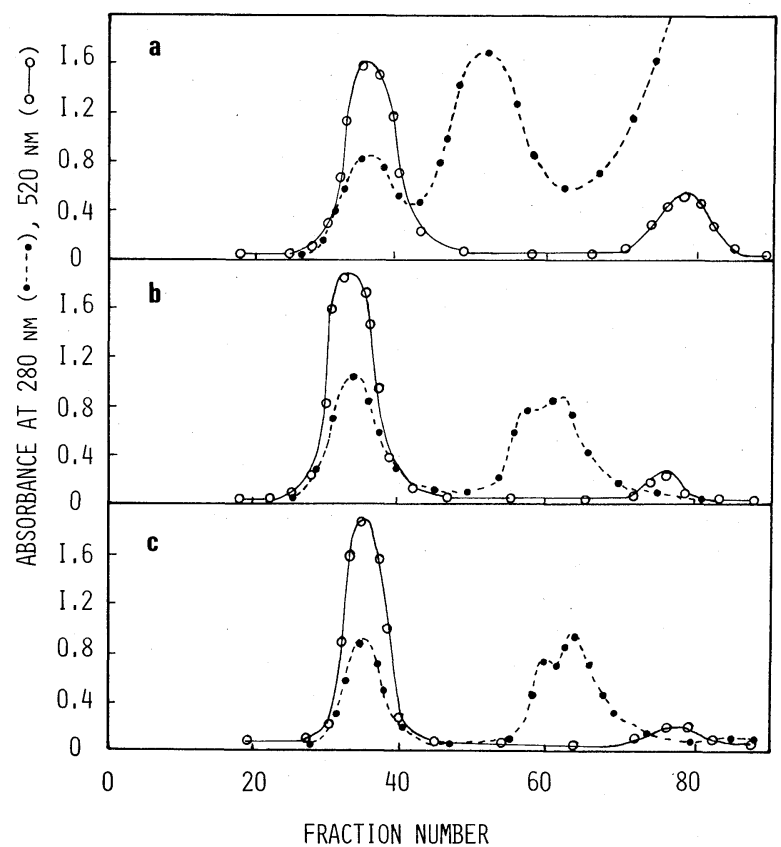

FIG. 1. Gel Filtration of the Proteolytic Digests of Ovomucin, Chalazae and Yolk Membrane on a Bio-Gel P-100 Column.

$\mathrm{a}, \mathrm{b}$ and $\mathrm{c}$ indicate ovomucin, chalazae and yolk membrane, respectively.

$\bigcirc-\bigcirc$, hexose (absorbance at $520 \mathrm{~nm}$ in the orcinol test);

, protein (absorbance at $280 \mathrm{~nm})$.

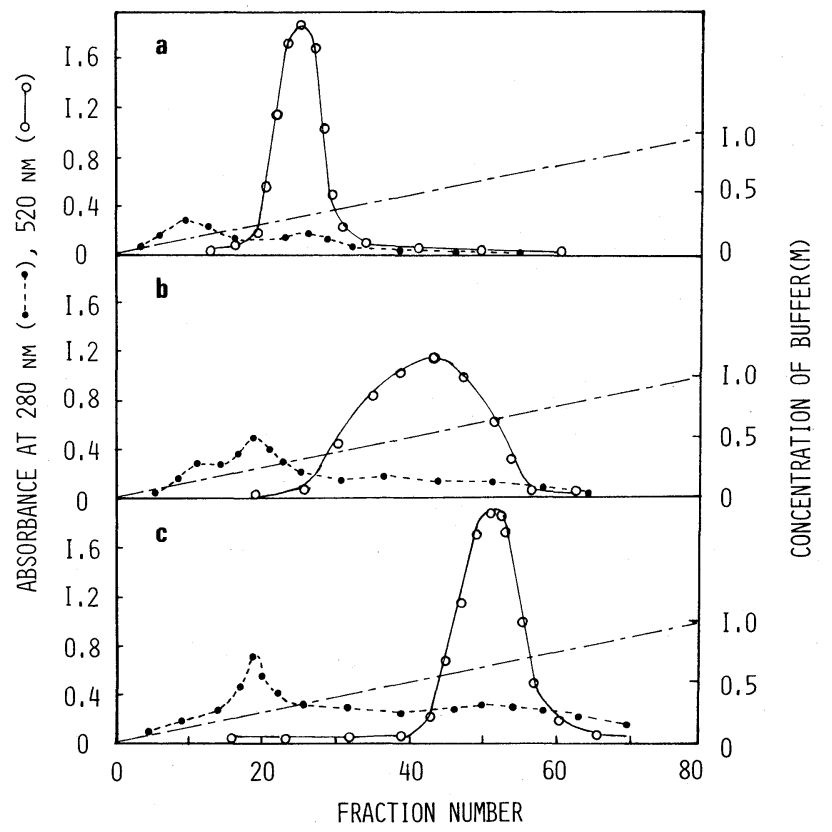

FIG. 2. Elution Profiles by a DEAE-Sephadex A-25 Column of Sulfated Glycopeptides in Ovomucin, Chalazae and Yolk Membrane.

$\mathrm{a}, \mathrm{b}$ and $\mathrm{c}$ indicate the sulfated glycopeptides in ovomucin, chalazae and yolk membrane, respectively.

$\mathrm{O}-\mathrm{O}$, hexose (absorbance at $520 \mathrm{~nm}$ in the orcinol test);

, protein (absorbance at $280 \mathrm{~nm}$ ). 
volume fraction. As shown in the previous paper, ${ }^{9)}$ the sulfated glycopeptide of ovomucin was eluted in the void volume fraction. It was confirmed from the analysis of sulfate residues in each glycopeptide fraction that the sulfated glycopeptides of chalazae and yolk membrane were also eluted in the void volume fraction.

The void volume fractions, sulfated glycopeptides, were further purified by a DEAESephadex A-25 column chromatography. Figure 2 shows the elution patterns by a DEAESephadex A-25 column of the sulfated glycopeptides in ovomucin, chalazae and yolk membrane, which eluted in that order with an increase in the concentration of $\mathrm{NaCl}$. Each elution profile was different, reflecting the differences in the contents of sialic acid and sulfate. The elution patterns of the sulfated glycopeptides in ovomucin and yolk membrane were single peak, while the elution pattern of those in chalazae was broad. The fractions, $20 \sim 30,30 \sim 55,43 \sim 58$, were separated as the sulfated glycopeptides of ovomucin, chalazae and yolk membrane, respectively, and the chemical compositions were analyzed.

Table II shows the carbohydrate composition of sulfated glycopeptides in ovomucin, chalazae and yolk membrane. These sulfated glycopeptides contained $\mathrm{N}$-acetylhexosamine $(23.2 \sim 26.8 \%)$, hexose $(23.6 \sim 24.4 \%)$, sialic acid $(11.2 \sim 18.0 \%)$, sulfate $(5.0 \sim 12.1 \%)$ and peptide $(17.5 \sim 18.1 \%)$. The carbohydrate compositions of these sulfated glycopeptides resembles that of the sulfated glycoproteins in epithelial mucins and gastric mucosa ${ }^{10)}$ except for the absence of fucose. The carbohydrate compositions of these sulfated glycopeptides were closely similar to each other, but the sulfate contents of glycopeptides in yolk membrane and chalazae were much higher than those in ovomucin. The molar ratios of sulfate to $N$-acetylhexosamine were $0.49,1.02$ and 1.12 in sulfated glycopeptides of ovomucin, chalazae and yolk membrane, respectively. The high ratio of sulfate to carbohydrates of glycopeptides in chalazae and yolk membrane suggests that these glycopeptides are compara-
Table II. Chemical Composition of SUlFATED GlyCOPEPTIDES IN Ovomucin, Chalazae AND Yolk Membrane

\begin{tabular}{lccc}
\hline & \multicolumn{2}{c}{ Contents $(\mathrm{g} / 100 \mathrm{~g}$ dry materials $)$} \\
\cline { 2 - 4 } & Ovomucin & Chalazae & $\begin{array}{c}\text { Yolk } \\
\text { membrane }\end{array}$ \\
& & & \\
\hline$N$-Acetyl- & & & \\
hexosamine & 23.3 & 26.8 & 23.6 \\
GlcNAc & 12.4 & 17.2 & 12.5 \\
GalNAc & 10.9 & 9.6 & 11.1 \\
Hexose & 24.2 & 24.4 & 23.6 \\
Gal & 21.8 & 24.4 & 23.6 \\
Man & 2.4 & 0 & 0 \\
Sialic acid & 18.0 & 14.6 & 11.2 \\
Sulfate & 5.0 & 12.1 & 11.8 \\
Protein & 18.0 & 18.1 & 17.5 \\
Sulfate & & & \\
\hline$N$-Acetyl- & 0.49 & 1.02 & 1.12 \\
hexosamine & & & \\
\hline
\end{tabular}

Table III. Amino ACID Composition of SUlFATED GlyCOPEPTIDES IN OVOMUCIN, Chalazae and Yolk Membrane

\begin{tabular}{lccc}
\hline & \multicolumn{3}{c}{ Contents (mol/100 mol) } \\
\cline { 2 - 4 } Amino acid & & & Yolk \\
& Ovomucin & Chalazae & $\begin{array}{c}\text { Yombrane } \\
\text { membs }\end{array}$ \\
\cline { 2 - 4 } Lys & 3.6 & 4.4 & 3.1 \\
His & 1.0 & 1.7 & 2.3 \\
Arg & 1.7 & 2.1 & 1.6 \\
Asp & $\mathrm{t}$ & 1.1 & 1.0 \\
Thr & 24.8 & 25.6 & 34.0 \\
Ser & 24.0 & 21.6 & 19.2 \\
Glu & 4.0 & 4.8 & 4.8 \\
Pro & 16.3 & 14.8 & 12.8 \\
Gly & $\mathrm{t}$ & 1.6 & 1.9 \\
Ala & 6.6 & 6.1 & 4.4 \\
Cys & 0 & 0 & 0 \\
Val & 4.4 & 4.7 & 4.5 \\
Met & 0.9 & 0.9 & 0.5 \\
Ile & 2.7 & 3.3 & 4.4 \\
Leu & 8.0 & 7.5 & 5.8 \\
Tyr & $\mathrm{t}$ & $\mathrm{t}$ & $\mathrm{t}$ \\
Phe & $\mathrm{t}$ & $\mathrm{t}$ & $\mathrm{t}$ \\
Trp & 0 & 0 & 0 \\
\hline & & & \\
\hline
\end{tabular}

$\mathrm{t}$, trace.

ble to the highest sulfated glycoproteins of those reported to date. ${ }^{10)}$

Table III shows the amino acid composition 


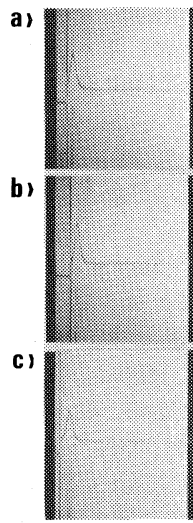

$10 \mathrm{~min}$
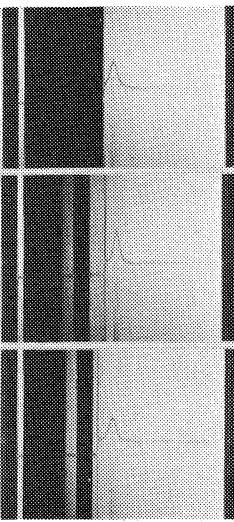

$20 \mathrm{~min}$
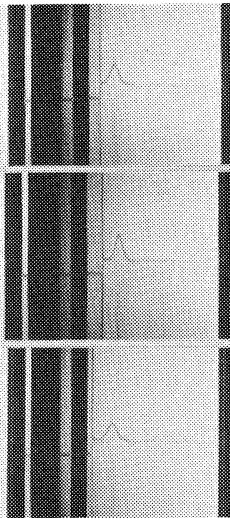

$30 \mathrm{~min}$

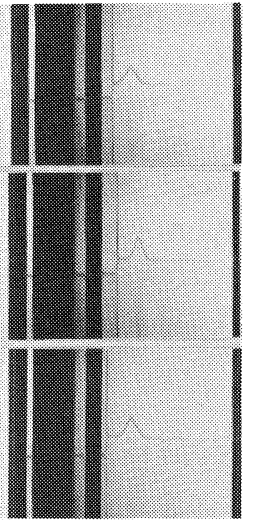

$40 \mathrm{~min}$

FIG. 3. Sedimentation Patterns of Sulfated Glycopeptides in Ovomucin, Chalazae and Yolk Membrane.

a), b) and c) indicate the sulfated glycopeptides in ovomucin, chalazae and yolk membrane, respectively.

of sulfated glycopeptides in ovomucin, chalazae and yolk membrane. The peptide portions of these sulfated glycopeptides were about 18 percent per dry weight, as well as the other sulfated glycoproteins reported in epithelial mucins and gastric mucosa. ${ }^{10)}$ The high contents of threonine, serine and proline are characteristic of sulfated glycoproteins, suggesting that the carbohydrate units are $O$ glycosidically linked to serine or threonine residues.

Figure 3 shows the sedimentation patterns of the sulfated glycopeptides in ovomucin, chalazae and yolk membrane. The sedimentation patterns of these sulfated glycopeptides exhibited a single peak. The sedimentation constants were $2.9,3.2$ and $3.2 \mathrm{~S}$ in the glycopeptides of ovomucin, chalazae and yolk membrane, respectively. This suggests that these sulfated glycopeptides are macromolecular, corresponding to proteins. The molecular weight of such a molecule, as sulfated glycoprotein contained lots of carbohydrates, is impossible to determine but approximate values can be calculated from the Scherage \& Mandelkern equation. ${ }^{11)}$ Using an assumed value of $2.15 \times 10^{6}$ for the $\beta$-function, the molecular weights of these sulfated glycopeptides were approximately $60,000 \sim 70,000$.

Although electrophoretic analysis was carried out by polyacrylamide gel electrophoresis,

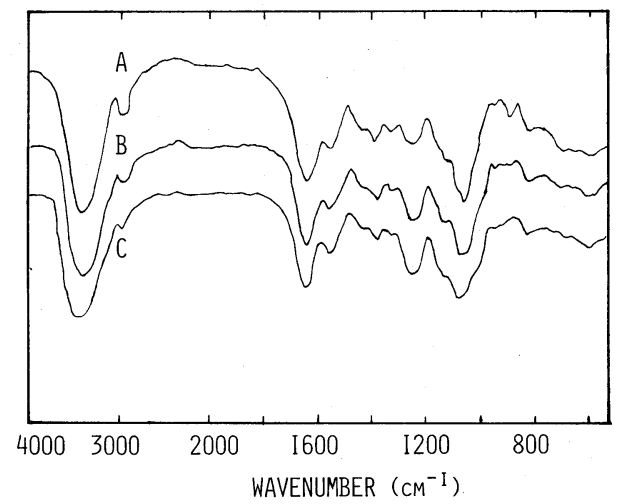

FIG. 4. Infrared Spectra of Sulfated Glycopeptides in Ovomucin, Chalazae and Yolk Membrane.

$\mathrm{A}, \mathrm{B}$ and $\mathrm{C}$ indicate the sulfated glycopeptides in ovomucin, chalazae and yolk membrane, respectively.

it was impossible to visualize the band in the gels stained with Coomassie Blue for proteins and Alcian Blue for carbohydrates. It may be that, because the glycopeptides contained lots of carbohydrates, they are difficult to immobilize in gels. Assuming from the DEAESephadex A-25 column chromatograms, the sulfated glycopeptides of ovomucin and yolk membrane are homogeneous but those of chalazae may be heterogeneous, despite the homogeneity with sedimentation pattern.

Figure 4 shows the infrared spectra of the sulfated glycopeptides in ovomucin, chalazae and yolk membrane. The infrared analysis 
indicated the presence of a sulfate ester with absorptions at $1240 \mathrm{~cm}^{-1} \quad(\mathrm{~S}=\mathrm{O}$ vibration) and $820 \mathrm{~cm}^{-1}$ (C-O-S vibration).

Thus, the presence of highly sulfated glycoproteins was confirmed in chalazae and yolk membrane. In addition, it was elucidated that chalazae and yolk membrane were not composed of ovomucin but other highly sulfated glycoproteins.

\section{REFERENCES}

1) R. E. Feeney, M. B. Rhodes and J. S. Anderson, J. Biol. Chem., 235, 2633 (1960).

2) Y. Sato and T. Kato, Poult. Sci., 59, 1416 (1980).

3) S. Kido, M. Janado and H. Nunoura, J. Biochem.,
81, 1543 (1977).

4) N. L. Allport, "Colorimetric Analysis," Vol. I, Chapman and Hall Ltd., 1957, p. 82.

5) A. Kato and Y. Sato, Agric. Biol. Chem., 36, 831 (1972).

6) O. W. Neuhaus and M. letzring, Anal. Chem., 29, 1230 (1957).

7) L. Warren, J. Biol. Chem., 234, 1971 (1959).

8) K. S. Dodgson and B. Spencer, Biochem. J., 55, 436 (1953).

9) A. Kato, S. Hirata, H. Sato and K. Kobayashi, Agric. Biol. Chem., 42, 835 (1978).

10) Z. Yoshizawa, "Glycoproteins," BBA Library, Vol. 5, ed. by A. Gottschalk, Elsvier Publishing Co., Amsterdam, 1972, p. 1000.

11) H. A. Scheraga and L. Mandelkern, J. Am. Chem. Soc., 75, 179 (1953). 